\title{
A NOTE ON COMPOUND SIMPLE GAMES
}

\section{T. PARTHASARATHY}

1. Introduction. A game is called simple if every coalition of players either wins or loses. Given any two simple games (players are assumed to be distinct in the two games) we define the product as one in which every winning coalition must include winning contingents from both the components. A typical example of a product game is any organization in which some member has veto power. L. S. Shapley has obtained the following theorem on product solutions [1].

THEOREM 1. For $i=1,2$, let $\left\{Y_{i}(\alpha) \mid 0 \leqq \alpha \leqq 1\right\}$ be a monotonic family of solutions of $\Gamma\left(P_{i}, W_{i}\right)$ except that $Y_{i}(1)$ need not be externally stable, and let $X_{i}(\alpha)=A_{P_{i}}-\operatorname{dom}_{i} Y_{i}(\alpha)$. Then

$$
X=\bigcup_{0 \leqq \alpha \leqq 1} X_{1}(\alpha) \times X_{\alpha} X_{2}(1-\alpha)
$$

is a solution of $\Gamma(P, W)=\Gamma\left(P_{1}, W_{1}\right) \otimes \Gamma\left(P_{2}, W_{2}\right)$.

In this connection L. S. Shapley has raised the following questions:

(1) Can the requirement of full monotonicity be relaxed outside a neighbourhood of $\alpha_{i}=1$ for the validity of Theorem 1 ?

(2) If the answer to (1) is "Yes," then does there exist a solution to product simple games which need not have the property of full monotonicity?

In $\S 3$, we give an affirmative answer to the first question, while in the last section, we present an example of a product solution which is not fully monotonic in the sense of Shapley.

The author is greatly indebted to Dr. L. S. Shapley for several highly helpful comments. Thanks are also due to the referee and to Mr. A. R. Padmanabhan for some useful suggestions and criticism.

\section{Definitions and notations.}

Simple games. We shall denote a simple game by the symbol $\Gamma(P, W)$ where $P$ is a finite set (players) and $W$ is a collection of subsets of $P$ (the winning coalitions). We demand that $P \in W$ and empty set not an element of $W$. Let $\Gamma\left(P_{1}, W_{1}\right)$ and $\Gamma\left(P_{2}, W_{2}\right)$ be two simple games with $P_{1} \cap P_{2}=\varnothing$ and let $P=P_{1} \cup P_{2}$. Then the product $\Gamma\left(P_{1}, W_{1}\right)$ $\otimes \Gamma\left(P_{2}, W_{2}\right)$ (for simplicity we will write $\left.P_{1} \otimes P_{2}\right)$ is defined as the game $\Gamma(P, W)$ where $W$ consists of all $S \subseteq P$ such that $S \cap P_{1} \in W_{1}$ and $S \cap P_{2} \in W_{2}$.

Received by the editors April 25, 1965. 
Imputations. Let $A_{P}$ denote the simplex of real nonnegative vectors $x$, such that $\sum x_{i}=1$. These vectors are traditionally called "imputations" where $P$ is the set of players in a simple game. Let us write $x(S)$ for $\sum_{i \in S} x_{i}$. Let $R_{S} x$ be the restriction of $x$ to $S$ thus:

$$
\begin{aligned}
R_{S} x & =x_{i} & & \text { if } i \in S, \\
& =0 & & \text { if } i \notin S .
\end{aligned}
$$

The barycentric projection of $x$ on $A_{S}$ is given by

$$
B_{S} x=\frac{1}{x(S)} R_{S} x
$$

This is well defined provided $x(S)>0$. Let $P_{1}$ and $P_{2}$ be fixed disjoint sets. Let $P=P_{1} \cup P_{2}$ and

$$
A_{P_{i}}=\left\{x \mid x \in A_{P} \text { and } x\left(P_{i}\right)=1\right\} .
$$

If $X$ and $Y$ are subsets of $A_{P_{1}}$ and $A_{P_{2}}$ respectively, we define an operation $X_{\alpha}$ by $(0 \leqq \alpha \leqq 1)$

$$
X \times \underset{\alpha}{\times}=\{z \mid z=\alpha x+(1-\alpha) y \text { for some } x \in X, y \in Y\} .
$$

We recall that a solution of the game $\Gamma(P, W)$ is a set $X$ of imputations such that $X=A_{P}-\operatorname{dom} X$ where $\operatorname{dom} X$ denotes the set of all $y \in A_{P}$ such that for some $x \in X$, the set $\left[i \mid x_{i}>y_{i}\right]$ is an element of $W$. The notation $\operatorname{dom}_{1}$ and $\operatorname{dom}_{2}$ will be used for domination w.r. to special classes $W_{1}, W_{2}$.

Definition. A parametrized family of sets of imputations

$$
[Y(\alpha): 0 \leqq \alpha \leqq 1]
$$

will be called semimonotonic if for every $\alpha, \beta, x$ such that $0 \leqq \alpha \leqq \beta \leqq 1$ and $x \in Y(\beta)$, there exists $y \in Y(\alpha)$ such that

$$
\alpha y \leqq \beta x .
$$

Definition. A semimonotonic family $\{Y(\alpha)\}$ is called fully monotonic if for every $\alpha, \beta, y$ such that $0 \leqq \alpha \leqq \beta \leqq 1$ and $y \in Y(\alpha)$, there exists $x \in Y(\beta)$ such that

$$
\alpha y \leqq \beta x .
$$

We will now generalize the concept of full monotonicity.

Definition. A semimonotonic family $Y(\alpha)$ is called $\partial$-monotonic $(0 \leqq \partial \leqq 1)$ if for every $\alpha, \beta, y$ such that $\partial \leqq \alpha \leqq \beta \leqq 1$ and $y \in Y(\alpha)$, there exists $x \in Y(\beta)$ such that $\alpha y \leqq \beta x$.

REMARK. Any $\partial$-monotonic family is necessarily $\partial_{1}$-monotonic if $\partial_{1} \geqq \partial$. 
3. On two theorems of Shapley. Shapley has proved Theorem 1 and the following theorem in [1].

THEOREM 2. Let $\left\{X_{i}(\alpha)\right\}$ be semimonotonic families of solutions of $\Gamma\left(P_{i}, W_{i}\right)$. Then

$$
X=\bigcup_{0 \leqq \alpha \leqq 1} X_{1}(\alpha) \underset{\alpha}{\times} X_{2}(\alpha)
$$

is a solution of $P_{1} \otimes P_{2}$.

The purpose of this section is to prove a theorem which includes both of these theorems.

Let $Y_{i}(\alpha) \subseteq A_{P_{i}}$ for $i=1,2$. Further suppose the families to be semimonotonic. Let $X_{i}(\alpha)=A_{P_{i}}-\operatorname{dom}_{i} Y_{i}(\alpha)$. For every $S_{j} \in W_{1}$ consider the following sets

$$
\begin{aligned}
\Lambda_{1}\left(S_{j}\right)=\{\alpha \mid 1>\alpha>0, & \text { there exists } x_{1} \in Y_{1}(\alpha) \\
& \text { and } \left.y_{1} \in X_{1}(1)-Y_{1}(1) \text { such that } \alpha x_{1}>y_{1} \text { on } S_{j}\right\} .
\end{aligned}
$$

Now choose one $\alpha_{j}$ from each $\Lambda_{1}\left(S_{j}\right)$ which is not empty. Let $\alpha_{0}$ $=\min \alpha_{j}$; we fix these $\alpha_{j}$ 's and it is clear that $\alpha_{0}>0$. We wish to remark that there is certain amount of arbitrariness in choosing $\alpha_{j}$ 's. Now we shall prove the following simple but useful lemma.

LEMMA 3. If $Y_{1}(\alpha)$ is $\alpha_{0}$-monotonic then there exist vectors $z_{j} \in Y_{1}(1)$ for those $j$ for which $\Lambda_{1}\left(S_{j}\right) \neq \varnothing$ and a $\partial_{1}$ with $0<\partial_{1} \leqq \alpha_{0}$ such that

$$
z_{j} \geqq \partial_{1} \text { on } S_{j} \text {. }
$$

Further if we take any $\alpha \in \Lambda_{1}\left(S_{j}\right), \alpha \geqq \partial_{1}$.

Proof. Since $\alpha_{j} \in \Lambda_{1}\left(S_{j}\right)$ there exists $x_{j} \in Y_{1}\left(\alpha_{j}\right)$ such that $\alpha_{j} x_{j}>0$ on $S_{j}$. Since $\alpha_{0} \leqq \alpha_{j}$ and the family is $\alpha_{0}$-monotonic there exists $z_{j}$ $\in Y_{1}(1)$ such that

$$
z_{j} \geqq \alpha_{j} x_{j} .
$$

Let $e_{j}$ denote the least component among the $S_{j}$ components of $x_{j}$. Define $\partial_{1}=\min \alpha_{j} e_{j}$. Then we have

$$
z_{j} \geqq \partial_{1} \text { on } S_{j} \text {. }
$$

Also it is trivial to check that $0<\partial_{1} \leqq \alpha_{0}$. Hence the first part of the proof of the lemma is complete. We shall now prove that any $\alpha \in \Lambda_{1}\left(S_{j}\right)$ will satisfy the inequality $\alpha \geqq \partial_{1}$.

Take any $\alpha \in \Lambda_{1}\left(S_{j}\right)$. This means there exists an $x_{1} \in Y_{1}(\alpha)$ and $y_{1} \in X_{1}(1)-Y_{1}(1)$ such that $\alpha x_{1}>y_{1}$ on $S_{j}$. We claim that at least one of the $S_{j}$ components of $y_{1}$ must be greater than or equal to $\partial_{1}$; 
otherwise $z_{j}>y_{1}$ on $S_{j}$ and therefore $y_{1} \in \operatorname{dom}_{1} z_{j} \subseteq \operatorname{dom}_{1} Y_{1}(1)$ which contradicts the assumption that $y_{1} \in X_{1}(1)=A_{P_{1}}-\operatorname{dom}_{1} Y_{1}(1)$. Therefore we have $\alpha>y_{1}\left(S_{j}\right) \geqq \partial_{1}$. Hence the proof of the lemma is complete.

Similarly we can get hold of a $\partial_{2}$ by constructing $\Lambda_{2}\left(S_{j}^{\prime}\right)$ for $S_{j}^{\prime} \in W_{2}$. If all the $\Lambda_{1}\left(S_{j}\right)$ and $\Lambda_{2}\left(S_{j}^{\prime}\right)$ are empty we define $\partial_{0}$ to be any positive number in the interval $(0,1]$-in fact we can take $\partial_{0}=1$. If $\Lambda_{1}\left(S_{j}\right) \neq \varnothing$ for some $j$ and $\Lambda_{2}\left(S_{j}^{\prime}\right) \neq \varnothing$ for some $j$ we define $\partial_{0}=\min \left[\partial_{1}, \partial_{2}\right]$. If $\Lambda_{1}\left(S_{j}\right) \neq \varnothing$ for some $j$ and $\Lambda_{2}\left(S_{j}^{\prime}\right)=\varnothing$ for all $j$, we define $\partial_{0}=\partial_{1}$ and in the other case we take $\partial_{0}=\partial_{2}$. In all these cases we see that $\partial_{0}>0$. Now we are in a position to state and prove our

THEOREM 4. Let $Y_{1}(\alpha)$ and $Y_{2}(\alpha)$ be a semimonotonic family of solutions to $\Gamma\left(P_{1}, W_{1}\right)$ and $\Gamma\left(P_{2}, W_{2}\right)$ respectively except that $Y_{1}(1)$ and $Y_{2}(1)$ need not be externally stable. Further suppose the family to be $\delta_{0}$ monotonic. Then

$$
X=\bigcup_{0 \leqq \alpha \leqq 1} X_{1}(\alpha) \underset{\alpha}{\times} X_{2}(1-\alpha)
$$

is a solution of $P_{1} \otimes P_{2}$, where $X_{2}(\alpha)=A_{P_{i}}-\operatorname{dom}_{i} Y_{i}(\alpha)$.

REMARK. Since $\left\{Y_{i}(\alpha)\right\}$ is semimonotonic it follows that $Y_{i}(1)$ is internally stable. If $Y_{i}(1)$ for $i=1,2$, is also externally stable, that is, if $Y_{2}(1)$ is a solution then it is not hard to check that $\Lambda_{1}\left(S_{j}\right)$ and $\Lambda_{2}\left(S_{j}^{\prime}\right)$ are all empty and we can take $\partial_{0}=1$. In other words Theorem 2 is included in our theorem. It is not hard to check that $\Lambda$-sets are empty if $Y_{i}(\alpha)$ are fully monotonic and hence Theorem 1 is also included in our theorem. We will show by giving an example that Theorem 4 is actually a generalization of Theorems 1 and 2 .

Proof of Theorem 4. Our proof follows along the same line as the one given for Theorem 2. Note that $X_{i}(\alpha)=Y_{i}(\alpha)$ for all $\alpha$ except $\alpha=1$.

External stability. Take any $y \in A_{P}$ and define $\beta_{\boldsymbol{i}}=y\left(P_{i}\right)$ and let $y_{i}$ be the barycentric projection of $y$ on $A_{P_{i}}$.

Case $1.0<\beta_{1}<1$.

Case 1a. $y_{1} \in X_{1}\left(\beta_{1}\right), y_{2} \in X_{2}\left(\beta_{2}\right)$, then $y=\beta_{1} y_{1}+\beta_{2} y_{2} \in X$.

Case 1b. $y_{1} \notin X_{1}\left(\beta_{1}\right), y_{2} \notin X_{2}\left(\beta_{2}\right)$, this means there exists $x_{1} \in Y_{1}\left(\beta_{1}\right)$, $x_{2} \in Y_{2}\left(\beta_{2}\right)$ such that $y_{i} \in \operatorname{dom}_{i} x_{i}$ for $i=1,2$.

Then the imputation defined by

$$
x=\beta_{1} x_{1}+\beta_{2} x_{2}
$$

which is in $X$, clearly dominates $y$ or $y \in \operatorname{dom} X$.

Case 1c. $y_{1} \in X_{1}\left(\beta_{1}\right), y_{2} \notin X_{2}\left(\beta_{2}\right)$.

Find $x_{2} \in Y_{2}\left(\beta_{2}\right)$ such that $y_{2} \in \operatorname{dom}_{2} x_{2}$. Let $x_{2}>y_{2}$ on $S_{2}^{\prime} \in W_{2}$. 
Choose $e>0$ so that $\beta_{2} x_{2}-\beta_{2} y_{2}>e$ on $S_{2}^{\prime}$. Using the semimonotonic property of $Y_{2}(\alpha)$ find $x_{2}^{\prime} \in Y_{2}\left(\beta_{2}-e\right)$ such that $\beta_{2} x_{2}-\left(\beta_{2}-e\right) x_{2}^{\prime}$ is nonnegative. This vector must be $\leqq e$ in all components, since no component of a nonnegative vector can exceed the sum of all components. If follows that we have

$$
\left(\beta_{2}-e\right) x_{2}^{\prime}>\beta_{2} y_{2} \text { on } S_{2}^{\prime} \text {. }
$$

Let $u_{1}$ be any interior point of $A_{P_{1}}$. Then the imputation $x$ defined by

$$
x=\beta_{1} y_{1}+e u_{1}+\left(\beta_{2}-e\right) x_{2}^{\prime}
$$

dominates $y$ on $P_{1} \cup S_{2}^{\prime}$. If $x \in X$ then $y \in \operatorname{dom} X$ and we are through. Suppose $x \notin X$. Then the barycentric projection of $x$ on $A_{P_{1}}$, namely

$$
x_{0}=\frac{\beta_{1}}{\beta_{1}+e} y_{1}+\frac{e}{\beta_{1}+e} u_{1}
$$

must not be an element of $X_{1}\left(\beta_{1}+e\right)=Y_{1}\left(\beta_{1}+e\right)$. Hence we can find $x_{1} \in Y_{1}\left(\beta_{1}+e\right)$ such that $x_{0} \in \operatorname{dom}_{1} x_{1}$. Then it is clear that the imputation $z$ defined by

$$
z=\left(\beta_{1}+e\right) x_{1}+\left(\beta_{2}-e\right) x_{2}^{\prime}
$$

dominates $y$. Since $z \in X$ it follows $y \in \operatorname{dom} X$.

Case 1 d. $y_{1} \notin X_{1}\left(\beta_{1}\right), y_{2} \in X_{2}\left(\beta_{2}\right)$ (like case $1 \mathrm{c}$ ).

Case 2. $\beta_{1}=0$.

Case 2a. $y_{2} \in X_{2}(1)$, then $y \in X$.

Case 2b. $y_{2} \notin X_{2}(1)$. This means $y_{2} \in \operatorname{dom}_{2} Y_{2}(1)$ and hence argument of case 1c can be repeated with the understanding that $\beta_{1} y_{1}=0$.

Case 3. $\beta_{1}=1$ (like case 2). This completes the proof of the external stability of $X$.

Internal stability of $X$. Suppose there exists $x, y \in X$ such that $x>y$ on $S \in W$. Let $S_{1}=S \cap P_{1}, S_{2}=S \cap P_{2} ; x=\alpha x_{1}+(1-\alpha) x_{2}$ and $y=\beta y_{1}$ $+(1-\beta) y_{2}$. Since $x>y$ on $S_{1} \cup S_{2}=S$, it follows that $0<\alpha<1$.

Case 1a. $1>\beta \geqq \alpha$. Since $\alpha>0, \beta$ is also strictly positive. Also $\alpha x_{1}>\beta y_{1}$ on $S_{1}$. Since $Y_{1}(\alpha)$ is semimonotonic, there exists $x_{1}^{\prime} \in Y_{1}(\alpha)$ such that $\beta y_{1} \geqq \alpha x_{1}^{\prime}$. Hence, $\alpha x_{1}>\alpha x_{1}^{\prime}$ on $S_{1}$ or $x_{1}>x_{1}^{\prime}$ on $S_{1}$ contradicting the internal stability of $Y_{1}(\alpha)$.

Case 1 b. $\alpha \geqq \beta>0$. This means $1>1-\beta \geqq 1-\alpha$. Since $\alpha \neq 1$ this case is similar to the previous case except that we have to utilize the semimonotonic property of $Y_{2}(\alpha)$.

Case 2a. $\beta_{1}=1$ that is $\alpha x_{1}>y=y_{1}$ on $S_{1}$. If $y_{1} \in Y_{1}(1)$ then using the semimonotonic property of $Y_{1}(\alpha)$ we will arrive at a contradiction. If $y_{1} \in X_{1}(1)-Y_{1}(1)$ then $\alpha \in \Lambda_{1}\left(S_{1}\right)$ and hence by Lemma $3, \alpha \geqq \partial_{1} \geqq \partial_{0}$. Since $Y_{1}(\alpha)$ is $\partial_{0}$-monotonic one can find $y_{1}^{\prime} \in Y_{1}(1)$ such that $y_{1}^{\prime} \geqq \alpha x_{1}$ 
$>y_{1}$. This implies $y_{1} \in \operatorname{dom}_{1} Y_{1}(1)$ or $y_{1} \notin X_{1}(1)$ which contradicts our assumption regarding $y_{1}$.

Case $2 \mathrm{~b} . \beta=0$ or $1-\beta=1$ and the argument can be carried over as in the case $2 \mathrm{a}$.

Hence the proof of our theorem is complete.

4. An example of a solution of product simple games. The following example shows that solutions to product simple games can be found that satisfy the conditions of Theorem 4 but not that of Theorems 1 or 2. In other words every solution that satisfies the conditions of Theorem 4 need not have the property of full monotonicity.

ExAmple. The four person game $J$ is defined by

$$
\Gamma(\overline{1234},\{\overline{124}, \overline{134}, \overline{234}, \overline{1234}\})
$$

where members in the curly brackets denote the winning coalitions of the game $J$.

$$
J=M_{3} \otimes B_{4} .
$$

$M_{3}$ denotes the 3-person simple majority game and $B_{4}$ the 1-person pure bargaining game. Define, for $0 \leqq \alpha \leqq 3 / 4$

$$
X_{1}(\alpha)=\bigcup_{0 \leqq \beta \leqq 1} Y(\beta)
$$

where

$$
Y(\beta)=\left\{\left(\beta^{2} / 2, \beta t, \beta(1-t)-\beta^{2} / 2,1-\beta\right) / 0 \leqq t \leqq 1-\beta / 2\right\}
$$

for $0 \leqq \beta<1$ and

$$
Y(1)=\{(1 / 2, t, 1 / 2-t, 0) / 0 \leqq t \leqq 1 / 2\} \cup(0,1 / 2,1 / 2,0) .
$$

Define for $3 / 4<\alpha \leqq \alpha_{0}$, where $\alpha_{0}$ is so chosen that

$$
\alpha_{0}\left(1-7 / 8\left(1+\alpha_{0}\right)\right)=\frac{1}{2} \text {, }
$$

the family:

$$
X_{1}(\alpha)=\bigcup_{0 \leqq \beta \leqq 1} Y(\beta)
$$

where

$$
\begin{aligned}
& Y(\beta)=\left\{\left(\frac{7}{8} \cdot \frac{\beta^{2}}{1+\alpha}, \beta t, \beta(1-t)-\frac{7}{8} \frac{\beta^{2}}{1+\alpha}, 1-\beta\right)\right. \\
& \text { where } \left.0 \leqq t \leqq 1-\frac{7 \beta}{8(1+\alpha)}\right\} \text { for } 0 \leqq \beta \leqq 1 .
\end{aligned}
$$

For $\alpha_{0}<\alpha<1$ define 


$$
X_{1}(\alpha)=\bigcup_{0 \leqq \beta \leqq 1} Y(\beta)
$$

where

$$
\begin{array}{r}
Y(\beta)=\left\{\left(\left(1-\frac{1}{2 \alpha}\right) \beta^{2}, \beta t, \beta(1-t)-\left(1-\frac{1}{2 \alpha}\right) \beta^{2}, 1-\beta\right)\right. \\
\text { where } \left.0 \leqq t \leqq 1-\left(1-\frac{1}{2 \alpha} \beta\right)\right\} \text { for } 0 \leqq \beta \leqq 1 .
\end{array}
$$

For $\alpha=1$ define $X_{1}(1)=\bigcup_{0 \leqq \beta \Sigma 1} Y(\beta)$ where

$$
Y(\beta)=\left\{\left(\beta^{2} / 2, \beta t, \beta(1-t)-\beta^{2} / 2,1-\beta\right) / 0 \leqq t \leqq 1-\beta / 2\right\} .
$$

Now it is not hard to check that the family $X_{1}(\alpha)$ is semimonotonic and that each $X_{1}(\alpha)$ is a solution to the game $J$ except $X_{1}(1)$ because the element $(0,1 / 2,1 / 2,0) \notin X_{1}(1) \cup \operatorname{dom}_{1} X_{1}(1)$. If $X_{1}^{\prime}(\alpha)=X_{1}(1)$ $\cup(0,1 / 2,1 / 2,0)$ then $X_{1}^{\prime}(1)$ is a solution to $J$. But $\left\{X_{1}(\alpha): 0 \leqq \alpha<1\right\}$ together with $X_{1}^{\prime}(1)$ is not semimonotonic for corresponding to $(0,1 / 2,1 / 2,0)$ there exists no element $x \in X_{1}(\alpha)$ for any $\alpha>3 / 4$ with $\alpha x \leqq(0,1 / 2,1 / 2,0)$. Further the family $\left\{X_{1}(\alpha): 0 \leqq \alpha \leqq 1\right\}$ is not fully monotonic because corresponding to the element $(0,1 / 2,1 / 2,0)$ $\in X_{1}(3 / 4)$ there exists no element $y \in X_{1}(1)$ with the property that

$$
y \geqq 3 / 4(0,1 / 2,1 / 2,0) .
$$

Hence it is clear that the conditions of Theorem 1 as well as Theorem 2 are violated. Moreover it is not difficult to check that $\Lambda_{1}\left(S_{j}\right)=\varnothing$ for all $j$ and as such we can take $\partial_{1}=1$. Hence this family $\left\{X_{1}(\alpha)\right\}$ can be used to produce product solution to arbitrary games of the form $J \otimes K$--solutions which Theorem 1 or Theorem 2 cannot predict.

\section{REFERENCE}

1. L. S. Shapley, Solutions of compound simple games, Annals of Mathematical Studies No. 52, pp. 267-305, Princeton Univ. Press, Princeton, N. J., 1964.

indian Statistical Institute, Calcutta 